\title{
Complicaciones asociadas al uso de aceite de silicona: revisión sistemática
}

\section{Complications associated with the use of silicone oil: A systematic review}

\author{
Andreas Di Luciano ${ }^{1 *}$, Agustina Adaniya1, Rosa Alvarado-Villacorta², Djorge Velickovich ${ }^{3}$, \\ Virgilio Morales-Cantón ${ }^{1}$ y Jose A. Roca ${ }^{4}$ \\ ${ }^{1}$ Departamento de Retina, Asociación para Evitar la Ceguera en México, I.A.P., Ciudad de México, México; ${ }^{2}$ Unidad de Investigación, Clínica Vista, \\ Lima, Perú; ${ }^{3}$ Fundación Visión, Asunción, Paraguay; ${ }^{4}$ Oftalmólogos Contreras, Lima, Perú
}

\section{Resumen}

Objetivo: Identificar y evaluar la información disponible sobre la frecuencia de las complicaciones tras el uso del aceite de silicona. Método: Se realizó una búsqueda sistemática en PubMed/Medline, Cochrane y Embase, hasta junio de 2020. Las listas de referencias de los estudios identificados y de las revisiones relevantes también fueron revisadas. Se incluyeron estudios en español e inglés, sin límite de fecha de publicación, de cualquier metodología, con una muestra mayor de 50 ojos, con pacientes con diagnóstico previo de desprendimiento de retina traccional, retinopatía diabética proliferativa, desgarros gigantes, desprendimiento de retina, por trauma, y pacientes con proliferación vitreorretiniana independientemente de la causa, con un seguimiento mínimo de 6 semanas. La evaluación de la calidad de los estudios se realizó según la clasificación del Centre for Evidence-Based Medicine. Resultados: Se incluyeron 13 estudios, la mayoría retrospectivos, con un nivel de evidencia 2b. La catarata (mediana: 90; rango intercuartílico [RIC]: 80-100\%), la emulsificación en la cámara anterior (mediana: 64.5; RIC: 46.5-82.25\%), la hipertensión ocular (mediana: 23.3; RIC: 22.15-35.1\%) y el redesprendimiento de retina (mediana: 22; RIC: 18.5-30\%) fueron las complicaciones más frecuentes, mientras que el bloqueo pupilar (mediana: 4.3; RIC: 3.8-4.8\%), el depósito conjuntival de aceite de silicona (mediana: 4.15; RIC: 3.75-4.57\%) y la hemorragia expulsiva (mediana: 1; RIC: 0.95-1.05\%) fueron las menos reportadas. Conclusiones: Las complicaciones más frecuentes en orden decreciente fueron cataratas, emulsificación en la cámara anterior, hipertensión ocular, redesprendimiento de retina, queratopatía, hipotonía, migración de aceite de silicona subretinal, resangrado, glaucoma secundario, pérdida visual inexplicable, bloqueo pupilar, depósitos conjuntivales de aceite de silicona y hemorragia expulsiva.

Palabras clave: Complicaciones por aceite de silicón. Retiro de aceite de silicón. Revisión sistemática.

\section{Abstract}

Purpose: To identify and assess the available information regarding the frequency of complications following the use of silicone oil (SO). Methods: A systematic search was conducted in PubMed/Medline, Cochrane and Embase, up to June 2020. The reference lists of identified studies and relevant reviews were also reviewed. We included studies in Spanish and English, with no publication date limits, of all types of methodology, with populations over 50 eyes, with patients previously diagnosed with tractional retinal detachment, proliferative diabetic retinopathy, giant retinal tears, traumatic retinal detachment and 
patients with proliferative vitreoretinopathy from any cause, with a minimum follow-up of 6 weeks. The quality assessment of the studies was conducted according to the Centre for Evidence-Based Medicine classification of levels of evidence. Results: $A$ total of 13 studies were included, most of which were retrospective, with a level of evidence $2 b$. Cataract (median: 90, interquartile range [IQR]: 80-100\%), silicone oil emulsification in the anterior chamber (median: 64.5; IQR: 46.5-82.25\%), ocular hypertension (median: 23.3; IQR: 22.15-35.1\%) and retinal redetachment (median: 22; IQR: 18.5-30\%) were the most frequent complications; while pupillary block (median: 4.3; IQR: 3.8-4.8\%), subconjunctival silicone oil deposits (median: 4.15; IQR: 3.75-4.57\%), and expulsive suprachoroidal hemorrhage (median: 1; IQR: 0.95-1.05\%) were the least frequent. Conclusions: The most frequent complications reported in decreasing order were cataracts, silicon oil emulsification in the anterior chamber, ocular hypertension, retinal redetachment, keratopathy, hypotonia, subretinal silicone oil migration, rebleeding, secondary glaucoma, unexplained visual loss, pupillary block, subconjunctival silicone oil deposits and expulsive suprachoroidal hemorrhage.

Key words: Silicon oil complications. Silicon oil removal. Systematic review.

\section{Introducción}

La aplicación de aceite de silicona (AS) en la cavidad vítrea se remonta a finales de los años 1950, cuando Stone ${ }^{1}$, en ojos de conejos, mostró que aceites de distintas viscosidades eran bien tolerados por un periodo de 2 años. El primer estudio en humanos fue realizado a comienzos de la década de 1960 por Cibis, et al. ${ }^{2}$, quienes usaron AS en 33 pacientes como taponamiento retiniano, demostrando una buena tolerancia retiniana por un periodo de 7 meses. Desde entonces, el uso de AS se prefiere para aquellos casos de alto riesgo de redesprendimiento de retina (reDR), como son los desprendimientos de retina complejos asociados a proliferación vitreorretiniana (PVR), los desgarros gigantes (DRG) y los desprendimientos de retina traccional (DRT) asociados a retinopatía diabética proliferativa (RDP), traumatismos o retinitis virales ${ }^{3}$.

El AS es una herramienta importante en la cirugía vitreorretiniana debido a que tiene la capacidad de desplazar el humor acuoso y mantener la adhesión retiniana; además, es un buen agente tampón en lesiones retinianas en ausencia de tracción. Si bien su utilización hoy en día se asocia con buenos resultados anatómicos, también se han descrito diversas complicaciones que pueden afectar casi todas las estructuras oculares e incluyen la formación de cataratas, hipertensión ocular (HTO), emulsificación en la cámara anterior, glaucoma secundario, reDR, hipotonía, bloqueo pupilar, migración subretinal del AS, pérdida visual inexplicable, depósitos subconjuntivales de AS, resangrado y hemorragia expulsiva ${ }^{3-30}$. La frecuencia de estas complicaciones varía considerablemente entre los diversos estudios, y pueden tener un gran impacto en la salud visual del paciente. Otra consideración importante en el uso del AS es la necesidad de un procedimiento quirúrgico adicional para su remoción una vez que se ha logrado la estabilización anatómica de la retina; sin embargo, hasta el día de hoy no existe un consenso en cuanto al tiempo adecuado de la remoción del AS basándose en las posibles complicaciones ${ }^{10-17}$. Teniendo en consideración la diversidad y la gravedad de las complicaciones reportadas, y a su vez la heterogeneidad de los reportes existentes, decidimos realizar una revisión sistemática para evaluar la frecuencia de las complicaciones tras el uso de AS.

\section{Método \\ Estrategia de búsqueda y selección de los estudios}

Se realizó una búsqueda sistemática en las bases de datos PubMed/Medline, Cochrane y Embase de documentos publicados hasta junio de 2020, utilizando las palabras clave "removal of silicone oil", "outcome of silicone oil removal" y "silicone oil complications". Adicionalmente, se revisó la lista de referencias de los estudios identificados y de las revisiones relevantes en el tema, buscando otros posibles estudios que pudieran ser incluidos.

Se incluyeron estudios de tipo ensayo clínico aleatorizado, estudios de cohortes y series de casos, con una muestra de más de 50 ojos, con pacientes con diagnóstico previo de DRT por RDP, DRG o por trauma, y pacientes con PVR independientemente de la causa, con un periodo mínimo de seguimiento de 6 semanas. Se excluyeron los estudios duplicados y con datos incompletos. Referente al idioma, se buscaron estudios en español y en inglés, sin limitación por fecha de publicación. La evaluación de la calidad de los estudios se realizó según la clasificación de los niveles de evidencia del Centre for Evidence-Based Medicine de Oxford (OCEBM). La selección de los estudios la 


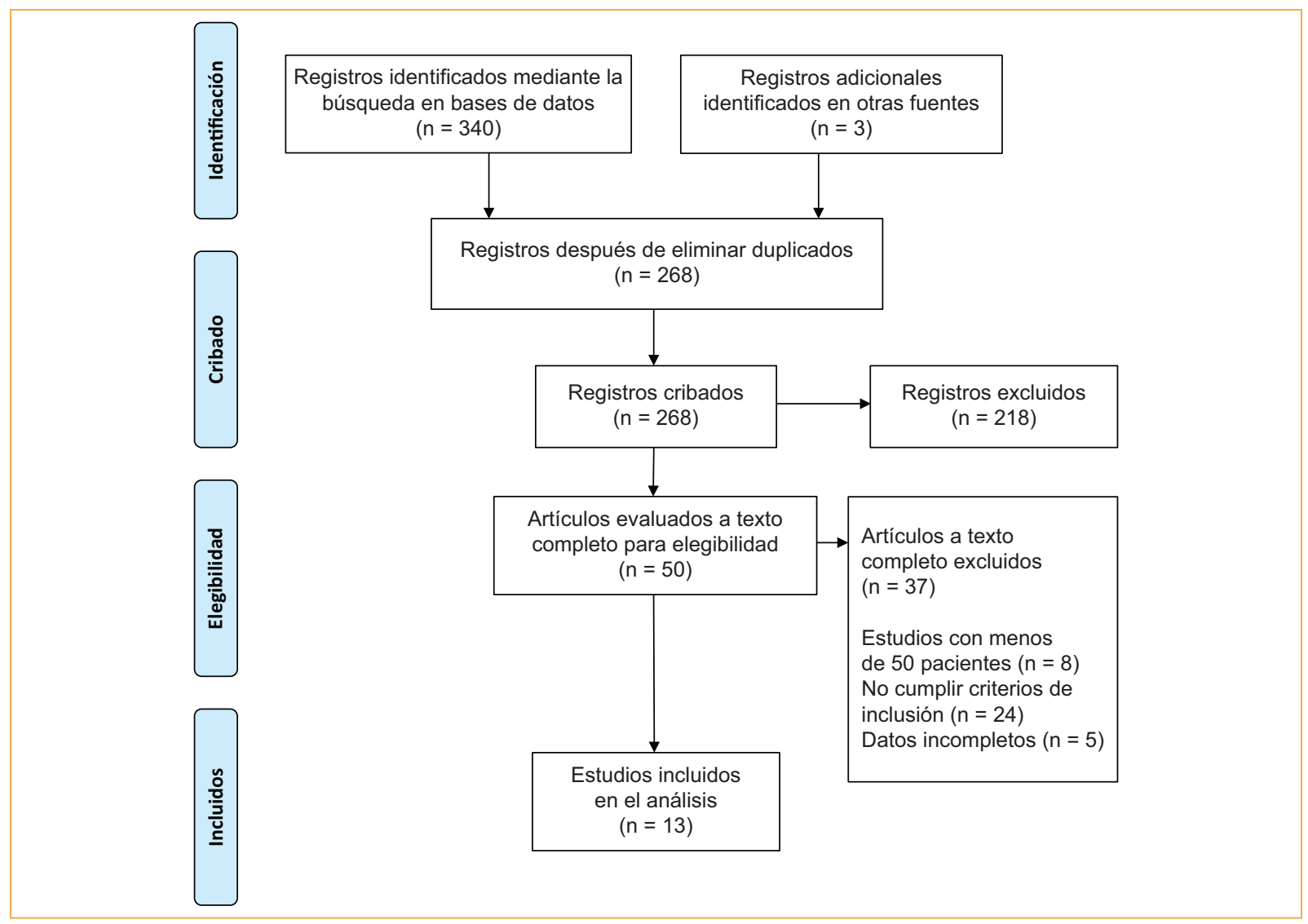

Figura 1. Diagrama de flujo para la inclusión de los estudios en la revisión sistemática según las recomendaciones PRISMA ${ }^{37}$.

hicieron de forma independiente dos investigadores (AD y DV) y las diferencias fueron resueltas por un tercer investigador $(\mathrm{AA})$.

\section{Extracción de datos y análisis}

Los estudios encontrados fueron exportados al software EndNote X9 para eliminar los artículos duplicados y subsecuentemente importados a Number v6.1 6369 para su tabulación. Los datos extraídos de los estudios fueron el autor principal, el año de publicación, el país de origen, la metodología, el número de pacientes, la edad, el sexo, el tipo de AS, las indicaciones más frecuentes, el intervalo de retiro del AS y las complicaciones encontradas.

Los datos fueron revisados por tres investigadores de forma independiente (AD, DV y $A A$ ). En caso de existir alguna observación sobre uno de los estudios, fue evaluado una vez más por otro investigador (JR). La revisión se evaluó una vez más por expertos en el tema (VM y JR) antes de crear el manuscrito final.
Los datos se agruparon en tablas descriptivas utilizando el software Numbers v6.1 6369 (Apple Inc.). Las variables categóricas se expresaron en porcentajes y los resultados numéricos como media y desviación estándar o como mediana y rango intercuartílico (RIC). Dada la heterogeneidad de los estudios, no se realizó metaanálisis.

\section{Resultados}

Con la estrategia de búsqueda se identificaron 343 estudios, de los cuales 75 fueron eliminados por duplicidad. Durante la revisión del título y del resumen se encontraron 50 estudios potenciales. Finalmente, tras la revisión del texto completo se incluyeron 13 artículos para la revisión (Fig. 1). La mayoría de los artículos correspondieron a reportes de los Estados Unidos de América y de Alemania; en todos los casos se utilizó AS de 1,000 o de 5,000 centistokes. Solo se identificó un ensayo clínico aleatorizado; la mayoría de los estudios fueron retrospectivos, con un nivel de evidencia $2 b$ según la clasificación del OCEBM. Las 
Tabla 1. Principales características de los estudios incluidos y niveles de evidencia según el Centre for Evidence-Based Medicine de 0xford (OCEBM) (n = 13)

\begin{tabular}{|c|c|c|c|c|c|c|c|c|c|c|}
\hline Estudios & País & Diseño & Población & Edad & Sexo & Tipo de AS & Indicaciones de cirugía & $\begin{array}{l}\text { Tiempo de } \\
\text { seguimiento }\end{array}$ & Intervalo de retiro AS & $\begin{array}{l}\text { Graduación } \\
\text { OCEBM }\end{array}$ \\
\hline $\begin{array}{l}\text { Casswell, } \\
\text { et al. } .^{10}(1987)\end{array}$ & Inglaterra & $\begin{array}{l}\text { Retrospectivo } \\
\text { (cohorte) }\end{array}$ & $\begin{array}{l}85 \text { ojos } \\
\text { (85 pacientes) }\end{array}$ & $\mathrm{NE}$ & NE & $\mathrm{NE}$ & $\begin{array}{l}\text { DRG: } 59(69.4 \%) \\
\text { PVR: } 26(30.58 \%)\end{array}$ & 18 meses & $\begin{array}{l}\mathrm{G} 1: \bar{X}=9.1 \text { (3-20 semanas) } \\
\mathrm{G} 2 \text { glaucoma: } \bar{X}=20.2 \\
\text { (3-60 semanas) } \\
\underline{\mathrm{G} 2} \text { queratopatía: } \\
\bar{X}=76(16-160 \text { semanas })\end{array}$ & $2 b$ \\
\hline $\begin{array}{l}\text { Lucke, et al. }{ }^{36} \\
\text { (1987) }\end{array}$ & Alemania & $\begin{array}{l}\text { Retrospectivo } \\
\text { (cohorte) }\end{array}$ & $\begin{array}{l}500 \text { ojos } \\
\text { (486 pacientes) }\end{array}$ & $46(1.5-85)$ & $\begin{array}{l}\text { H: } 311(62 \%) \\
\text { M: } 189(38 \%)\end{array}$ & $5000 \mathrm{cSt}$ & $\begin{array}{l}\text { PVR: } 55 \% \\
\text { DRG: } 15 \% \\
\text { Rupturas posteriores: } 14.6 \% \\
\text { RDP + DRT: } 18 \% \\
\text { Trauma: } 7.9 \%\end{array}$ & 15.7 meses & $6-18$ meses & $2 b$ \\
\hline $\begin{array}{l}\text { Federman y } \\
\text { Schubert }{ }^{15} \\
\text { (1988) }\end{array}$ & $\begin{array}{l}\text { Estados } \\
\text { Unidos }\end{array}$ & $\begin{array}{l}\text { Retrospectivo } \\
\text { (cohorte) }\end{array}$ & $\begin{array}{l}150 \text { ojos } \\
\text { (170 pacientes) }\end{array}$ & 40-60 años & NE & $\mathrm{NE}$ & $\begin{array}{l}\text { PVR C3 o peor: } 68.66 \% \\
\text { RDP + DRT: } 21.33 \% \\
\text { DRG: } 5.3 \%\end{array}$ & $\begin{array}{c}\bar{X}=31.6 \\
\text { meses }(6 \\
\text { meses-5 } \\
\text { años) }\end{array}$ & 3-6 meses & $2 b$ \\
\hline $\begin{array}{l}\text { Riedel, et al. }{ }^{31} \\
\text { (1990) }\end{array}$ & Alemania & $\begin{array}{l}\text { Retrospectivo } \\
\text { (cohorte) }\end{array}$ & $\begin{array}{l}415 \text { ojos } \\
\text { (415 pacientes) }\end{array}$ & NE & NE & $5000 \mathrm{cSt}$ & $\begin{array}{l}\text { PVR por DR: } 49 \% \\
\text { PVR por trauma: } 13 \% \\
\text { RDP + DRT: } 38 \%\end{array}$ & 30 meses & $6-30$ meses & $2 b$ \\
\hline $\begin{array}{l}\text { Franks y } \\
\text { Leaver }{ }^{11} \\
\text { (1991) }\end{array}$ & Alemania & $\begin{array}{l}\text { Retrospectivo } \\
\text { (cohorte) }\end{array}$ & $\begin{array}{l}120 \text { ojos } \\
\text { (116 pacientes) }\end{array}$ & $41.5(12-81)$ & NE & $1000 \mathrm{cSt}$ & $\begin{array}{l}\text { DRG: } 47.5 \% \\
\text { Roturas posteriores: } 13.3 \% \\
\text { PVR: } 38.33 \%\end{array}$ & 5 años & $\bar{X}=30$ (3 semanas -5 años) & $2 b$ \\
\hline $\begin{array}{l}\text { Silicon Study } \\
\text { Group }^{16} \\
\text { (1992-1994) }^{\text {(1994 }}\end{array}$ & $\begin{array}{l}\text { Estados } \\
\text { Unidos }\end{array}$ & ECA & $\begin{array}{l}101 \text { ojos ( } \\
101 \text { pacientes) }\end{array}$ & $66.2(24-84)$ & $\begin{array}{l}\text { H: } 17(33 \%) \\
\text { M: } 30(67 \%)\end{array}$ & $1000 \mathrm{cSt}$ & DRR + PVR $(100 \%)$ & 36 meses & 8 semanas- 6 meses & $1 b$ \\
\hline $\begin{array}{l}\text { Scholda, } \\
\text { et al. }{ }^{17}(2000)\end{array}$ & Austria & $\begin{array}{l}\text { Prospectivo } \\
\text { (cohorte) }\end{array}$ & $\begin{array}{l}112 \text { ojos } \\
\text { (106 pacientes) }\end{array}$ & $\begin{array}{c}\text { Grupo DR: } \\
44.2 \pm 21.4 \\
\text { Grupo no DR: } \\
50.9 \pm 19.5\end{array}$ & NE & $\begin{array}{l}1000 \mathrm{cSt} \\
(\mathrm{n}=26) \\
5000 \mathrm{cSt} \\
(\mathrm{n}=86)\end{array}$ & $\begin{array}{l}\text { PVR }(n=59), \operatorname{RDP}(n=34) \\
\text { Agujero macular, CEIO, } \\
\text { OVCR, uveítis, hemangioma } \\
\text { retinal }(n=19)\end{array}$ & $\begin{array}{l}7 \text { días- } 6.9 \\
\text { años }\end{array}$ & $\begin{array}{l}\text { Grupo DR: } 185 \pm 94.5 \text { días } \\
\text { Grupo no DR: } 319 \pm 235 \\
\text { días }\end{array}$ & $1 b$ \\
\hline $\begin{array}{l}\text { Falkner, } \\
\text { et al. }{ }^{12}(2001)\end{array}$ & Austria & $\begin{array}{l}\text { Retrospectivo } \\
\text { (cohorte) }\end{array}$ & $\begin{array}{l}115 \text { ojos } \\
\text { (115 pacientes) }\end{array}$ & $54.9(13-83)$ & NE & $5000 \mathrm{cSt}$. & $\begin{array}{l}\text { DRR + PVR: } 89.2 \% \\
\text { DRT: } 5.4 \% \text {; trauma } 5.4 \%\end{array}$ & $\begin{array}{l}6 \text { meses-3.5 } \\
\text { años }\end{array}$ & 13.3 meses & $2 b$ \\
\hline $\begin{array}{l}\text { Moya, et al. }{ }^{20} \\
(2015)\end{array}$ & Inglaterra & $\begin{array}{l}\text { Retrospectivo } \\
\text { (cohorte) }\end{array}$ & $\begin{array}{l}421 \text { ojos } \\
\text { (421 pacientes) }\end{array}$ & $53.1(40-74)$ & $\begin{array}{l}\text { PVI (14) } \\
\text { H: } 12(86 \%) \\
\text { M: } 2(24 \%)\end{array}$ & 1300 cSt & $\begin{array}{l}\text { Desgarro gigante: } 71.4 \% \\
\text { PVR: } 28.5 \%\end{array}$ & 2 años & 141 días (76-218) & $2 b$ \\
\hline
\end{tabular}


Tabla 1. Principales características de los estudios incluidos y niveles de evidencia según el Centre for Evidence-Based Medicine de 0xford (OCEBM) ( $n=13$ ) (Continuación)

\begin{tabular}{|c|c|c|c|c|c|c|c|c|c|c|}
\hline Estudios & País & Diseño & Población & Edad & Sexo & Tipo de AS & Indicaciones de cirugía & $\begin{array}{l}\text { Tiempo de } \\
\text { seguimiento }\end{array}$ & Intervalo de retiro AS & $\begin{array}{l}\text { Graduación } \\
\text { OCEBMM }\end{array}$ \\
\hline $\begin{array}{l}\text { Anaya-Pava, } \\
\text { et al. }{ }^{7}(2017)\end{array}$ & México & $\begin{array}{l}\text { Retrospectivo } \\
\text { (cohorte) }\end{array}$ & $\begin{array}{l}269 \text { ojos } \\
\text { ( } 235 \text { pacientes) }\end{array}$ & $\begin{array}{c}H: 58.6(26-86) \\
\text { M: } 59.7 \\
(24-88)\end{array}$ & $\begin{array}{l}\text { H: } 139(51.7 \%) \\
\text { M: } 130 \\
(48.3 \%)\end{array}$ & $5000 \mathrm{cSt}$ & $\begin{array}{l}\text { DRT: } 65 \% \\
\text { DRR + PVR: } 32 \% \\
\text { Trauma: } 3 \%\end{array}$ & 5 años & $\begin{array}{l}11 \text { meses }(1-58) \\
\text { Trauma: } \bar{X}=11 \text { (3-24 } \\
\text { semanas) } \\
\text { RDP: } \bar{X}=10 \text { (5-16 semanas) } \\
\text { DRR + VRP }=\bar{X} 11 \\
\text { (8-19 semanas) }\end{array}$ & $2 b$ \\
\hline $\begin{array}{l}\text { Roca, et al. } .^{18} \\
\text { (2017) }\end{array}$ & $\begin{array}{l}\text { Argentina } \\
\text { Brasil } \\
\text { Costa Rica } \\
\text { Colombia } \\
\text { Estados } \\
\text { Unidos } \\
\text { España } \\
\text { México } \\
\text { Puerto Rico } \\
\text { Venezuela }\end{array}$ & $\begin{array}{l}\text { Retrospectivo, } \\
\text { multicéntrico } \\
\text { (cohorte) }\end{array}$ & $\begin{array}{l}324 \text { ojos } \\
\text { (324 pacientes) }\end{array}$ & $\begin{array}{c}\text { PVI: } \\
49.2 \pm 16.4 \\
(16-73)\end{array}$ & $\begin{array}{l}\text { PVI (19) } \\
\text { H: } 8(42 \%) \\
\text { M: } 11(68 \%)\end{array}$ & $\begin{array}{c}1000 \mathrm{cSt} \\
(42.1 \%) \\
5000 \mathrm{cSt} \\
(57.9 \%)\end{array}$ & $\begin{array}{l}\text { DBT: } 47.4 \% \\
\text { Desgarro gigante: } 10.5 \% \\
\text { DRT diabético: } 47.4 \% \\
\text { PVR: } 31.6 \% \\
\text { DR mácula off: } \\
63.2 \%\end{array}$ & 14.8 meses & $14.8 \pm 4,4$ meses & $2 b$ \\
\hline $\begin{array}{l}\text { Jabbour, } \\
\text { et al. }{ }^{13} \text { (2018) }\end{array}$ & Líbano & $\begin{array}{l}\text { Prospectivo } \\
\text { (cohorte) }\end{array}$ & $\begin{array}{l}254 \text { ojos } \\
\text { (254 pacientes) }\end{array}$ & $\begin{array}{c}55.33 \pm 16.29 \\
(8-86)\end{array}$ & H: $175(68.9 \%)$ & $\begin{array}{l}1000 \mathrm{cSt} \\
(\mathrm{n}=152) \\
5000 \mathrm{cSt} \\
(\mathrm{n}=102)\end{array}$ & $\begin{array}{l}\text { DRT: } 61.1 \% \\
\text { PVR: } 38.9 \%\end{array}$ & $\begin{array}{c}22.4 \pm 18.1 \\
(6-76 \text { meses })\end{array}$ & 6 meses & $1 b$ \\
\hline $\begin{array}{l}\text { Abdalkader, } \\
\text { et al. }{ }^{32} \text { (2019) }\end{array}$ & $\begin{array}{l}\text { Estados } \\
\text { Unidos }\end{array}$ & $\begin{array}{l}\text { Retrospectivo } \\
\text { (cohorte) }\end{array}$ & $\begin{array}{l}57 \text { ojos } \\
\text { (57 pacientes) }\end{array}$ & $\begin{array}{l}\text { Migración } \\
56.1 \pm 16.6 \\
\text { No migración } \\
57.5 \pm 18.6\end{array}$ & $\begin{array}{l}\text { H: } 31(54.4 \%) \\
\text { M: } 26(45.6 \%)\end{array}$ & $\begin{array}{c}1000 / \\
5000 \mathrm{cSt}\end{array}$ & $\begin{array}{l}\text { DRT: } 59,6 \% \text { Trauma: } 7 \% \\
\text { Retinitis por CMV: } 5.2 \% \\
\text { Glaucoma: } 3.5 \% \\
\text { Hemorragia posoperatoria: } \\
1.75 \% \\
\text { Infección congénita: } 1.75 \% \\
\text { Desconocido: } 21 \%\end{array}$ & $\begin{array}{c}1 \text { mes a } 4 \\
\text { años tras } \\
\text { inyección de } \\
\text { AS }\end{array}$ & NA & $2 b$ \\
\hline
\end{tabular}

AS: aceite de silicona; CEIO: cuerpo extraño intraocular; CMV: citomegalovirus; cSt: centistokes; DBT: desprendimiento de retina traccional diabético; DR: desprendimiento de retina; DRG: desgarro de retina gigante; DRR: desprendimiento de retina regmatógeno; DRT: desprendimiento de retina traccional; ECA: ensayo clínico aleatorizado; G1: retiro como prevención de complicaciones; G2: retiro como tratamiento de complicaciones; H: hombres; M: mujeres; NA: no aplica; NE: no especifica; OVCR: oclusión de la vena central de la retina; PVI: pérdida visual inexplicable; PVR: proliferación vitreorretiniana; RDP: retinopatía diabética proliferativa. 
Tabla 2. Frecuencia de complicaciones tras el uso de aceite de silicona

\begin{tabular}{|l|c|}
\hline Complicación & Mediana (RIC) \\
\hline Hipertensión ocular & $23.3(22.15-35.1 \%)$ \\
\hline Glaucoma secundario & $6(4.5-9.1 \%)$ \\
\hline Bloqueo pupilar & $4.3(3.8-4.8 \%)$ \\
\hline Cataratas & $90(80-100 \%)$ \\
\hline Queratopatía & $6.3(4.55-13.9 \%)$ \\
\hline Redesprendimiento & $22(18.5-30 \%)$ \\
\hline Hipotonía & $13.5(9.55-17.65 \%)$ \\
\hline Hemorragia expulsiva & $1(0.95-1.05 \%)$ \\
\hline Pérdida visual inexplicada & $4.6(3.95-5.25 \%)$ \\
\hline Migración submacular & $8.95(7.42-10.47 \%)$ \\
\hline Resangrado & $6.1(5.25-6.85 \%)$ \\
\hline Depósito subconjuntival de aceite & $4.15(3.75-4.57 \%)$ \\
\hline Emulsificación en cámara anterior & $64.5(46.75-82.25 \%)$ \\
\hline RIC: rango intercuartílico. & \\
\hline
\end{tabular}

RIC: rango intercuartílico.

características de los estudios incluidos en el análisis se resumen en la tabla 1.

Las complicaciones tras el uso de AS reportadas se describen en la tabla 2, y las más frecuentes fueron cataratas con una mediana de 90 (RIC: 80-100\%), emulsificación en la cámara anterior con una mediana de 64.5 (RIC: $46.5-82.25 \%$ ), HTO con una mediana de 23.3 (RIC: $22.15-35.1 \%$ ) y reDR con una mediana de 22 (RIC: $18.5-30 \%$ ). Por otro lado, entre las menos frecuentes figuran bloqueo pupilar con una mediana de 4.3 (RIC: 3.8-4.8\%), depósito subconjuntival del AS con una mediana de 4.15 (RIC: $3.75-4.57 \%$ ) y finalmente hemorragia expulsiva con una mediana de 1 (RIC: 0.95-1.05\%).

\section{Discusión}

Es conocido que la mayoría de las indicaciones para el uso de AS son desprendimientos de retina complejos, como PVR, DRG, RDP, DRT o debido a trauma o infección viral ${ }^{1-25}$. En estos casos, el AS es de gran utilidad para buscar aplicar la retina y disminuir el riesgo de reDR; sin embargo, se ha estudiado ampliamente que el uso de este tampón puede asociarse a varias complicaciones que hay que tener en consideración al hacer el seguimiento de estos pacientes ${ }^{10-17}$.
Una de las complicaciones más frecuentes es la formación de catarata, que puede producirse de novo o a partir de una opacidad preexistente ${ }^{18}$. La formación de cataratas se ve en el $100 \%$ en algunas series a 5 años de seguimiento ${ }^{8}$, y la incidencia está relacionada con el tiempo de duración del AS en el ojo. Los estudios ultraestructurales han demostrado alteraciones en las células epiteliales del cristalino ${ }^{7-9}$. La catarata es también causa de baja visión y debe considerarse su extracción cuando se planifique el retiro del AS, teniendo en consideración el recuento de células endoteliales corneales, el cual podría estar alterado por el efecto del $\mathrm{AS}^{23-34}$. En nuestra revisión encontramos que esta es la principal complicación reportada. Su dispersión es amplia y está directamente relacionada con el tiempo que se mantuvo el AS en la cavidad, ya que hay estudios que hacen seguimiento a 5 años y otros con menor intervalo ${ }^{18}$.

También se han reportado como complicaciones frecuentes el glaucoma secundario y la HTO, con unas tasas del $3-7.8 \%$ para el glaucoma ${ }^{7-10}$ y del $11-46.6 \%$ para la $\mathrm{HTO}^{10-22}$. Los factores de riesgo asociados son pseudofaquia, afaquia, alta miopía, HTO previa y el uso de AS de baja viscosidad ${ }^{12}$. También se han descrito casos de bloqueo pupilar, los cuales deben de ser manejados con iridotomía periférica inferior con láser Nd-YAG o reabriendo una iridotomía preexistente ocluida. Muchos de estos pacientes mejoran al retirar el AS, pero el $6.8 \%$ necesitarán cirugía filtrante para controlar la presión intraocular ${ }^{12}$. Es importante, previo a la cirugía con AS, advertir a los pacientes sobre la posibilidad de desarrollo de HTO y, por lo tanto, la necesidad de tratamiento tópico prolongado, o incluso procedimientos quirúrgicos si esta complicación continúa en el posoperatorio ${ }^{12}$. Nuestro estudio muestra la alta frecuencia de esta complicación, y su amplia dispersión se correlaciona en parte con la metodología empleada para definir la HTO en los distintos trabajos y con el tratamiento que recibieron los pacientes.

Las tasas de reDR varían ampliamente, entre el $14 \%^{14}$ y el $50 \%{ }^{15}$; esta variación depende de las características del desprendimiento de retina y de la patología de base. La mayoría de los estudios, y casi todos de naturaleza retrospectiva, describen que la mayor tasa de reDR se produce en los primeros días posoperatorios. El factor más asociado al reDR es la presencia o la formación de PVR; cuanto mayor y más anterior sea la PVR, mayor es el riesgo de reDR. Las otras causas de reDR son la RDP con DRT, los DRG y los asociados a traumatismos ${ }^{16-19}$. Al analizar los valores encontrados en la literatura observamos una gran dispersión en cuanto a esta complicación (RIC: 18.5-30\%). Cabe 
mencionar que el reDR se ha estudiado en forma conjunta o aislada más que otras complicaciones, teniendo en consideración la indicación quirúrgica de estos pacientes (desprendimientos complejos), lo que también colabora a su expresión numérica como una de las principales complicaciones asociadas.

La hipotonía tras el retiro del AS se descrito en el $5.2-22.5 \%$ de los $\operatorname{casos}^{21}$. Los estudios demuestran que ocurre con mayor frecuencia en pacientes posoperados de desprendimiento de retina con compromiso macular, y el mecanismo no solo dependería de la causa de la cirugía, sino que también estaría asociado a una alteración del cuerpo ciliar y a la inflamación en la cámara anterior ${ }^{21}$.

La pérdida visual inexplicada es un desafío clínico, ya que muchos pacientes luego del retiro del AS pueden tener una agudeza visual mejor corregida inferior a la esperada sin causa anatómica demostrable. Se han descrito cada vez más casos asociados y su causa no es del todo comprendida ${ }^{25-29}$. Los mecanismos patogénicos sugeridos son disfunción macular directa producto del contacto del AS, alteraciones en factores de crecimiento y fototoxicidad foveal ${ }^{4-25,28}$. Muchos autores creen que la HTO podría estar asociada, pero no existen estudios con metodología de peso para afirmar ninguna de estas teorías. Las tasas van del $3.3 \%$ al $5.9 \%$ de los casos $^{15-32}$.

Teniendo en consideración toda la información disponible sobre las posibles complicaciones del uso de AS y su asociación con el tiempo de permanencia, surge la pregunta sobre el momento apropiado para el retiro del AS; sin embargo, no existe hasta el día de hoy un consenso establecido, ya que muchos de estos pacientes tienen patologías complejas y de curso impredecible. Si bien el AS es un excelente tamponador retiniano a largo plazo, su uso debe de ser provisional debido a las complicaciones que surgen por su uso prolongado. Un evento importante es la emulsificación del AS; a mayor duración intraocular, mayor probabilidad de emulsificación ${ }^{15-35}$. Un estudio encontró que el tiempo promedio para que empiece la emulsificación del AS tras la cirugía es de 13 meses $^{33}$. La evidencia disponible no permite responder a la pregunta sobre el tiempo óptimo para el retiro del AS, por lo que se sugiere que debe de ser individualizado en cada paciente; no obstante, la mayoría de los autores coinciden en un mínimo de 3 a 6 meses de taponamiento ${ }^{12-33}$.

Referente a las limitaciones del estudio, debido a la heterogeneidad de los artículos incluidos no fue posible realizar un metaanálisis para consolidar la información disponible. Producto de nuestros criterios de inclusión y metodología de estudio, se pueden haber omitido otros trabajos relevantes, por lo que para minimizar esto se realizó una evaluación por tres autores por separado y luego en conjunto.

En conclusión, las complicaciones más frecuentes reportadas fueron, en orden decreciente, cataratas, emulsificación en la cámara anterior, HTO, reDR, queratopatía, hipotonía, migración de AS subretinal, resangrado, glaucoma secundario, pérdida visual inexplicable, bloqueo pupilar, depósitos conjuntivales de AS y hemorragia expulsiva.

\section{Conflicto de intereses}

Los autores declaran no tener ningún conflicto de intereses.

\section{Responsabilidades éticas}

Protección de personas y animales. Los autores declaran que para esta investigación no se han realizado experimentos en seres humanos ni en animales.

Confidencialidad de los datos. Los autores declaran que han seguido los protocolos de su centro de trabajo sobre la publicación de datos de pacientes.

Derecho a la privacidad y consentimiento informado. Los autores declaran que en este artículo no aparecen datos de pacientes.

\section{Bibliografía}

1. Stone W. Alloplasty in Surgery of the Eye. New Engl J Med. 1958;258:486.

2. Cibis PA, Becker B, Okun E, Cannan S. The use of liquid silicone in retinal detachment surgery. Arch Ophthalmol. 1962;68:590-9.

3. Gallemore RP, McCuen BW II. Silicone oil in vitreoretinal surgery. En: Ryan SJ, editor. Retina. 4th ed. Philadelphia: Elsevier Mosby; 2006. p. 2211-34.

4. Barca F, Caporossi T, Rizzo S. Silicone oil: different physical proprieties and clinical applications. Biomed Res Int. 2014;2014:502143.

5. Joussen AM, Wong D. The concept of heavy tamponades-chances and limitations. Graefes Arch Ophthalmol. 2008;246:1217-24.

6. Ryan SJ. Silicone oils: physicochemical properties. En: Rayan SJ, editor. Retina. 4th ed. Philadelphia: Elsevier Mosby; 2006. p. 2191-210.

7. Anaya-Pava EJ, Sáenz-Bocanegra CH, Baeza García JA, Rodríguez V. Estudio retrospectivo de complicaciones asociadas al aceite de silicona. Experiencia de 5 años en un hospital público del norte de México. Rev Mex Oftal. 2017;91:76-81.

8. Zhu L, Zhao K, Lou D. Apoptosis factors of lens epithelial cells responsible for cataractogenesis in vitrectomized eyes with silicone oil tamponade. Med Sci Monit. 2016;22:788-96

9. Soliman W, Sharaf M, Abdelazeem K, El-Gamal D, Nafady A. Ultrastructural effects of silicone oil on the clear crystalline lens of the human eye. Eur J Ophthalmol. 2018;28:566-72.

10. Casswell AG, Gregor ZJ. Silicone oil removal. II. Operative and postoperative complications. Br J Ophthalmol. 1987;71:898-902.

11. Franks WA, Leaver PK. Removal of silicone oil - rewards and penalties. Eye (Lond). 1991;5(Pt 3):333-7.

12. Falkner $\mathrm{Cl}$, Binder $\mathrm{S}$, Kruger $\mathrm{A}$. Outcome after silicone oil removal. $\mathrm{Br} \mathrm{J}$ Ophthalmol. 2001;85:1324-7.

13. Jabbour E, Azar G, Antoun J, Kourie HR, Abdelmassih Y, Jalkh A. Incidence and risk factors of ocular hypertension following pars plana vitrectomy and silicone oil injection. Ophthalmologica. 2018;240:129-34.

14. Hutton WL, Azen SP, Blumenkranz MS, Lai MY, McCuen BW, Han DP, et al. The effects of silicone oil removal. Silicone Study Report 6. Arch Ophthalmol. 1994;112:778-85. 
15. Federman JL, Schubert HD. Complications associated with the use of silicone oil in 150 eyes after retina-vitreous surgery. Ophthalmology. 1988;95:870-6.

16. The Silicone Study Group. Vitrectomy with silicone oil or sulfur hexafluoride gas in eyes with severe proliferative vitreoretinopathy: results of a randomized clinical trial. Silicone Study Report 1. Arch Ophthalmol. 1992;110:770-9.

17. Scholda C, Egger S, Lakits A, Walch K, von Eckardstein E, Biowski R. Retinal detachment after silicone oil removal. Acta Ophthalmol Scand. 2000;78:182-6.

18. Roca JA, Wu L, Berrocal M, Rodriguez F, Alezzandrini A, Alvira G et al. Un-explained visual loss following silicone oil removal: results of the Pan American Collaborative Retina Study (PACORES) Group. Int J Retina Vitreous. 2017:3:26.

19. Scheerlinck LM, Schellekens PA, Liem AT, Steijns D, van Leeuwen R Incidence, risk factors, and clinical characteristics of unexplained visua loss after intraocular silicone oil for macula-on retinal detachment. Retina. 2016;36:342-50.

20. Moya R, Chandra A, Banerjee PJ, Tsouris D, Ahmad N, Charteris DG. The incidence of unexplained visual loss following removal of silicone oil. Eye (Lond). 2015;29:1477-82

21. Winter M, Eberhardt W, Scholz C, Reichenbach A. Failure of potassium siphoning by Müller cells: a new hypothesis of perfluorocarbon liquid-induced retinopathy. Invest Ophthalmol Vis Sci. 2000;41:256-61.

22. Christensen UC, La Cour M. Visual loss after use of intraocular silicone oil associated with thinning of inner retinal layers. Acta Ophthalmol. 2012:90:733-7.

23. Szaflik JP, Kmera-Muszy『ska M. Confocal microscopy imaging of the cornea in patients with silicone oil in the anterior chamber after vitreoretinal surgery. Graefes Arch Clin Exp Ophthalmol. 2007;245:210-4

24. Legler U, Seiberth V, Knorz MC, Jung E. [Loss of corneal endothelial cells following pars plana vitrectomy and silicone oil implantation]. Fortschr Ophthalmol. 1990;87:290-3.
25. Kuhn F, Kover F, Szabo I, Mester V. Intracranial migration of silicone oil from an eye with optic pit. Graefes Arch Clin Exp Ophthalmol. 2006;244:1360-2.

26. Michel G, Meyer L, Naoun O. [Sudden visual loss following silicone oil removal: three patients treated for giant retinal tear]. J Fr Ophtalmol. 2009;32:104-11.

27. Winter M, Eberhardt W, Scholz C, Reichenbach A. Failure of potassium siphoning by Müller cells: a new hypothesis of perfluorocarbon liquid-induced retinopathy. Invest Ophthalmol Vis Sci. 2000;41:256-61.

28. Asaria $\mathrm{RH}$, Kon $\mathrm{CH}$, Bunce C, Sethi CS, Limb GA, Khaw PT, et al. Silicone oil concentrates fibrogenic growth factors in the retro-oil fluid. $\mathrm{Br} \mathrm{J}$ Ophthalmol. 2004;88:1439-42.

29. Cazabon S, Groenewald C, Pearce IA, Wong D. Visual loss following removal of intraocular silicone oil. Br J Ophthalmol. 2005;89:799-802.

30. Jiang F, Krause M, Ruprecht KW, Hille K. Risk factors for anatomical success and visual outcome in patients undergoing silicone oil removal. Eur J Ophthalmol. 2002;12:293-8.

31. Riedel KG, Gabel VP, Neubauer L, Kampik A, Lund OE. Intravitreal silicone oil injection: complications and treatment of 415 consecutive patients. Graefes Arch Clin Exp Ophthalmol. 1990;228:19-23.

32. Abdalkader M, Takumi K, Chapman MN, Barest GD, Peeler C, Sakai O. Subretinal and retrolaminar migration of intraocular silicone oil detected on CT. AJNR Am J Neuroradiol. 2019;40:1557-61.

33. Toklu Y, Cakmak HB, Ergun SB, Yorgun MA, Simsek S. Time course of silicone oil emulsification. Retina. 2012;32:2039-44

34. Satchi K, Bolton A, Patel CK. Loss of vision once silicone oil has been removed. Retina. 2005;25:807-8.

35. Miller JB, Papakostas TD, Vavvas DG. Complications of emulsified silicone oil after retinal detachment repair. Semin Ophthalmol. 2014;29:312-8.

36. Lucke $\mathrm{KH}$, Foerster $\mathrm{MH}$, Laqua $\mathrm{H}$. Long-term results of vitrectomy and silicone oil in 500 cases of complicated retinal detachments. Am J Ophthalmol. 1987; 104:624-33.

37. Moher D, Liberati A, Tetzlaff J, Altman DG; PRISMA Group. Preferred reporting items for systematic reviews and meta-analyses: the PRISMA Statement. PLoS Med. 2009;6:e1000097. 